\title{
Estudo da Influência de Resíduos Catalíticos na Degradação de Plásticos Reciclados (Blenda HDPE/PP e PET) Provenientes de Lixo Urbano
}

\author{
Amélia S. F. Santos, José Augusto M. Agnelli e Sati Manrich
}

\begin{abstract}
Resumo: A degradação derivada do processamento, uso, descarte, lavagem e reprocessamento foram caracterizados neste estudo. Para o HDPE/PP pós-consumo, a degradação foi estudada em função da presença de garrafas de HDPE/LDPE reprocessadas (recicladas) e da presença de tampas e rótulos de LDPE e PP. Esses materiais mostraram uma influência significativa na degradação total de HDPE/PP apenas quando presentes em conjunto. Os resíduos que apresentaram menor estabilidade termo-oxidativa foram os rótulos e tampas de LDPE e PP. As análises usadas na caracterização da influência destes resíduos foram espectroscopia do Infra-vermelho (IR) e Calorimetria Diferencial Exploratória (tempo de indução oxidativa - OIT). Além disso, a degradação do PET foi estudada em função das condições de lavagem e da presença de resíduos de soda cáustica e adesivo. Foram usadas medidas de viscosidade intrínseca e de titulação para caracterizar estas variáveis. Foi observado que apesar do uso de altas temperaturas $\left(80^{\circ} \mathrm{C}\right)$ durante a lavagem, a extensão de tempo desta etapa (5 ou $15 \mathrm{~min}$ ) não afetou a degradação do PET, pelo menos não em níveis detectáveis pelas análises. No entanto, a remoção do resíduo de adesivo mostrou uma influência significativa na degradação catalítica do PET.
\end{abstract}

Palavras-chave: Plásticos reciclados, degradação, lixo urbano, blenda HDPE/PP, PET.

\section{Introdução}

Desde que os plásticos conquistaram o mercado de embalagens, sua fração nos resíduos sólidos urbanos tornou-se significativa. Preocupados com o incremento exponencial deste resíduo ao longo do tempo, visto sua baixa biodegradabilidade, iniciaram-se vários estudos visando seu reaproveitamento.

A reciclagem surgiu como uma das frentes de estudo mais promissoras para este fim. Mundial- mente, deste então, este procedimento tem sido empregado e estudado. No entanto, esses resíduos plásticos pós-consumo, antes de serem reciclados já sofreram algum tipo de deterioração de suas propriedades físicas e químicas. Estas reações de degradação normalmente são oriundas da polimerização, processamento, uso e descarte do polímero.

Na região de estudo, cidade do interior de São Paulo com cerca de 200.000 habitantes, os plásticos rígidos presentes em maior proporção, no 
resíduo sólido urbano, são o PET, o HDPE e o PP. Estes por sua vez, apresentam características de degradação diferenciadas entre si. As poliolefinas caracterizam-se por serem sensíveis à presença de traços de metais pesados provenientes de impurezas, pigmentos, resíduos de catalisadores ou de equipamentos empregados no processamento, além de também apresentar sensibilidade a resíduos ácidos (cloro do $\mathrm{TiCl}_{3}, \mathrm{AIR}_{2} \mathrm{Cl}$ ou $\mathrm{MgCl}_{2}$ ), cargas, antiestáticos, pigmentos e resíduos de hidroperóxidos gerados em processamentos anteriores. Mais especificamente, o PP apresenta uma menor estabilidade oxidativa comparado ao HDPE, devido à sua maior proporção de hidrogênios ligados a carbonos terciários ${ }^{[1]}$. Estes pontos, juntamente como a vizinhança de insaturações, são considerados como sendo os pontos mais vulneráveis das diferentes estruturas químicas poliméricas durante os processos termooxidativos de degradação. ${ }^{[1,2]}$

Já o PET é sensível a resíduos de PVC e de adesivos e à presença de umidade nas temperaturas de processamento. Os resíduos de PVC e adesivos são problemas característicos do reprocessamento de garrafas de PET. Os adesivos, apesar de poderem ser desprezados em processos de inserção de rótulos tipo camisa, ainda são usados para colar rótulos nas garrafas, principalmente nas garrafas verdes. Já os resíduos de PVC se tornam comuns em reciclagens de grande escala, devido à competição de mercado entre estes dois polímeros, dificultando sua diferenciação por aplicação. Tanto o PVC, quanto os adesivos, geram compostos ácidos nas temperaturas de processamento (ácido hidroclórico e acético, respectivamente), que catalisam a hidrólise dos grupos éster ${ }^{[3]}$. Ainda durante a reciclagem de resíduos de PET, podem-se utilizar soluções alcalinas a altas temperaturas, para lavagem dos mesmos e, neste caso, as ligações éster podem ser quebradas por catálise básica. Porém, independente do tipo de catálise que possa prevalecer, a degradação térmica e autooxidativa adicionam-se à degradação hidrolítica, durante o reprocessamento. Consequentemente, há um aumento no teor de grupos finais carboxílicos paralelamente a uma diminuição na massa molar dos poliésteres $^{[2,4]}$.

É neste contexto que se insere este trabalho, pois busca-se estimar a influência de resíduos de LDPE e PP, dos rótulos e tampas das garrafas de HDPE e de resíduos já anteriormente reciclados, no processo de degradação da blenda HDPE/PP. Além disto, avaliou-se a influência dos resíduos de adesivo, nas proporções encontradas, e de diferentes condições de lavagem na degradação do PET pós-consumo.

\section{Experimental}

\section{Materiais}

Os resíduos de plásticos rígidos, $\mathrm{HDPE}, \mathrm{PP}$ e PET, foram coletados na Usina de Separação e Compostagem de Lixo Urbano de Araraquara -SP. As embalagens de PET consistiram predominantemente de garrafas de bebidas carbonatadas, com embalagens de água e óleo esporádicas. Já as de HDPE e PP são basicamente de produtos de limpeza, higiene pessoal, utensílios, alimentos e óleos automotivos.

O resíduo de PET utilizado, compôs-se de uma mistura de 53,3\% de PET verde com $46,7 \%$ de PET incolor. A composição utilizada durante os ensaios para as poliolefinas foi uma mistura de $90 \%$ de HDPE e $10 \%$ de PP. Estas proporcões estão de acordo com as encontradas no lixo ${ }^{[5]}$.

Os aditivos de limpeza utilizados foram soda caústica em escama, da A. R. I. Chemicals do Brasil Ltda. e umectante Erkantol NR-BD, da Bayer S/A

\section{Métodos}

A lavagem das garrafas de PET e de poliolefinas foi realizada em 3 etapas: pré-lavagem com água $\left(23^{\circ} \mathrm{C}\right)$; lavagem com diferentes soluções e enxágüe com água a $23^{\circ} \mathrm{C}$. As condições da etapa de lavagem foram: $0,5 \%$ de $\mathrm{NaOH}$ e $0,3 \%$ de Erkantol em água a $80^{\circ} \mathrm{C}$, para o PET; $1 \%$ de $\mathrm{NaOH}$ e $0,3 \%$ de Erkantol em água a $23^{\circ} \mathrm{C}$, para as poliolefinas. As etapas de pré-lavagem e enxágüe foram realizadas num sistema de tambor rotativo, enquanto a etapa de lavagem foi efetuada num tanque com rotor lateral. Estas condições foram determinadas como adequadas em estudos preliminares. Algumas variações em termos de tempo de enxágüe e lavagem foram realizadas para o PET, visando determinar a influência de resíduos de adesivo e de soda cáustica. Estas condições estão descritas na Tabela $1 \mathrm{com}$ as respectivas denominações para cada amostra. 
Tabela 1. Identificação das amostras de PET.

\begin{tabular}{lcc}
\hline \multicolumn{1}{c}{ Condição de lavagem* } & Descrição da amostra \\
\hline Lavagem e enxágüe por 5 min. & PET com soda e com adesivo. \\
Lavagem por 15 min e enxágüe por 5 min. & PET com remoção parcial de adesivo e com soda. \\
Lavagem por 5 min e enxágüe por 10 min. & PET sem soda e com adesivo. \\
$\begin{array}{l}\text { Garrafas sem adesivos (apenas com rótulo tipo } \\
\text { "hot melt") lavadas e enxaguadas por } 5 \text { min. }\end{array}$ & PET com soda e sem adesivo. \\
\hline
\end{tabular}

* As condições de pré-lavagem e a concentração de soda cáustica e aditivo não foram variadas.

Como aproximadamente $17 \%$ das embalagens de HDPE são de água sanitária e este produto é uma blenda de LDPE com HDPE, além de em algumas empresas ser feito à base de produto reciclado, optou-se por estudar sua influência como agente catalisador da degradação da blenda HDPE/PP. Outro fator que também representa $6 \%$ do total, é a presença de rótulos e tampas que são em sua maioria de PP e LDPE, materiais mais susceptíveis à degradação que o HDPE. Para estudar a influência destes resíduos nas amostras de HDPE/PP, estas foram separadas de acordo com a Tabela 2.

Devido à diversidade das amostras, estas foram homogeneizadas após a lavagem, através de um processo de mistura por agitação; moídas na forma de pó, homogeneizadas novamente para, então, realizar as caracterizações.

A secagem padrão adotada tanto para os "flakes" de HDPE e PP antes do processamento, quanto para o pó, foi de 28 horas a $50^{\circ} \mathrm{C}$, em estufa com circulação de ar. Já a secagem de PET para o processamento, foi feita por 24 horas a $45^{\circ} \mathrm{C}$, em estufa de circulação de ar, seguida por 6 horas a $160^{\circ} \mathrm{C}$, em estufa a vácuo. Para as análises, o pó de PET foi seco por 4 horas a $65^{\circ} \mathrm{C}$, em estufa de circulação de ar.

$\mathrm{Na}$ extrusão, utilizou-se uma extrusora Gerst de $24 \mathrm{~mm}$ diâmetro e $\mathrm{L} / \mathrm{D}=24$, a $100 \mathrm{rpm}$. O perfil de

Tabela 2. Denominação das amostras de HDPE analisadas.

\begin{tabular}{ll}
\hline \multicolumn{1}{c}{ Nome da amostra de HDPE } & Código \\
\hline $\begin{array}{l}\text { Sem garrafa de água sanitária e sem } \\
\text { Rótulos e tampas }\end{array}$ & HDPE/PP ss \\
$\begin{array}{l}\text { Apenas garrafas de água sanitária e sem } \\
\text { rótulos e tampas } \\
\text { Resíduo total sem rótulos e tampas }\end{array}$ & HDPE/PP cs \\
Resíduo total com rótulos e tampas & HDPE/PP tc \\
\hline
\end{tabular}

temperatura empregado foi de 150,170 e $180^{\circ} \mathrm{C}$ para o HDPE/PP e de 210,235 e $245^{\circ} \mathrm{C}$ para o PET.

Os espectros de infravermelho foram obtidos num Espectrômetro de Infravermelho (FTIR) Perkin-Elmer-Spectrum 1000. A determinação da quantidade de carbonila nas diferentes amostras de HDPE foi feita em triplicata, utilizando-se a razão entre as áreas dos picos em 1740 e $1895 \mathrm{~cm}^{-1}$.

A determinação do tempo de indução oxidativa (OIT), por Calorimetria Diferencial de Varredura (DSC), foi realizada no analisador DSC modulado Du Pont modelo 2920. O procedimento adotado está de acordo com a ASTM D 3895 - 95.

As medidas de viscosidade intrínseca foram feitas em fenol/tetracloroetano (60:40), segundo a norma ASTM D 4603 - 91. Seu cálculo foi feito pela equação de Billmeyer.

A determinação de grupos finais carboxílicos das amostras de PET foi feita em duplicata segundo metódo de Polh ${ }^{6}$. Na realização das medidas, utilizou-se uma microbureta de $5 \mathrm{ml}$ com sensibilidade de $0,01 \mathrm{ml}$.

\section{Resultados e Discussão}

Os resultados das análises de infravermelho e OIT, da blenda HDPE/PP, antes e após o processamento na extrusora de rosca simples, estão apresentados nas Tabelas 3 e 4, respectivamente. Uma melhor visualização destes dados está ilustrada nas Figuras 1 e 2.

Como pode ser visto, a amostra de HDPE/PP que não possui resíduos de LDPE e nem PP adicionais (HDPE ss) foi a que apresentou um maior índice de carbonila, enquanto a amostra com maior teor de LDPE e PP (HDPE/PP tc) apresentou um índice relativamente menor. Isto pode indicar que o HDPE em estudo no geral encontra-se inici- 
Tabela 3. Valores dos índices de carbonila para as amostras de HDPE/PP.

\begin{tabular}{ccc}
\hline \multirow{2}{*}{ Amostra } & \multicolumn{2}{c}{ Área $_{\mathbf{1 7 4 0}} /$ Área $_{\mathbf{1 8 9 5}}$} \\
\cline { 2 - 3 } & $\begin{array}{c}\text { Antes do } \\
\text { reprocessamento }\end{array}$ & $\begin{array}{c}\text { Depois do } \\
\text { reprocessamento }\end{array}$ \\
\hline HDPE/PP ss & 0,45 & 0,46 \\
HDPE/PP cs & 0,24 & 0,30 \\
HDPE/PP ts & 0,28 & 0,32 \\
HDPE/PP tc & 0,24 & 0,39 \\
\hline
\end{tabular}

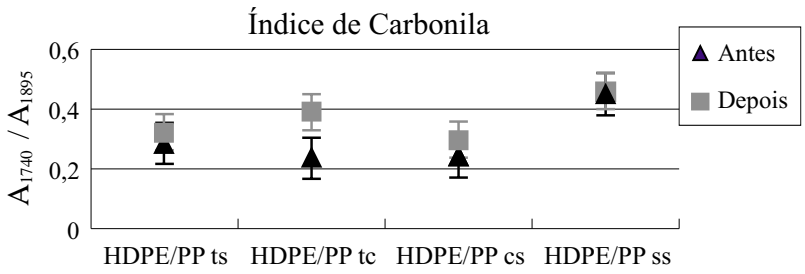

Figura 1. Gráfico da variação do índice de carbonila (Area ${ }_{1740} / \operatorname{Area}_{1895}$ ) da mistura de "flakes" HDPE/PP antes e após o reprocessamento.

almente com um nível de degradação maior que os resíduos de LDPE e PP. No entanto após o reprocessamento, a amostra de HDPE/PP sem possíveis resíduos catalíticos praticamente não aumentou seu índice de carbonila, indicando que esta amostra praticamente não degradou durante o reprocessamento.

Pode-se observar ainda que a contribuição conjunta dos resíduos de LDPE e PP de tampas e rótulos de garrafas, juntamente com as garrafas reprocessadas de HDPE/LDPE fez com que houvesse um incremento significativo na absorção, da amostra HDPE/PP tc, na região da carbonila, podendo indicar a ação desses resíduos como catalisadores da degradação da amostra de HDPE/ PP. Isoladamente, o incremento no índice de carbonila destas amostras não foi significativo devido ao mesmo estar dentro do erro do experimento, 0,06 .

Com os dados de OIT pode-se confirmar a menor estabilidade oxidativa dos resíduos de PP e LDPE adicionais, visto a maior concentração de hidrogênios ligados a carbonos terciários nestes polímeros. Uma redução na estabilidade oxidativa das amostras, após um passo de extrusão, não foi possível ser detectada. Porém, pode-se concluir que as tampas e rótulos de LDPE e PP possuem uma estabilidade menor que as garrafas reprocessadas de água sanitária. Isto devido a sua alta influência sob o tempo de
Tabela 4. Medidas do Tempo de Indução Oxidativa (OIT) das amostras de HDPE/PP.

\begin{tabular}{ccc}
\hline \multirow{2}{*}{ Amostra } & \multicolumn{2}{c}{ OIT (min) } \\
\cline { 2 - 3 } & $\begin{array}{c}\text { Antes do } \\
\text { reprocessamento }\end{array}$ & $\begin{array}{c}\text { Depois do } \\
\text { reprocessamento }\end{array}$ \\
\hline HDPE/PP ss & 3,67 & 3,89 \\
HDPE/PP cs & 3,59 & 3,51 \\
HDPE/PP ts & 4,09 & 4,10 \\
HDPE/PP tc & 3,46 & 3,45 \\
\hline
\end{tabular}

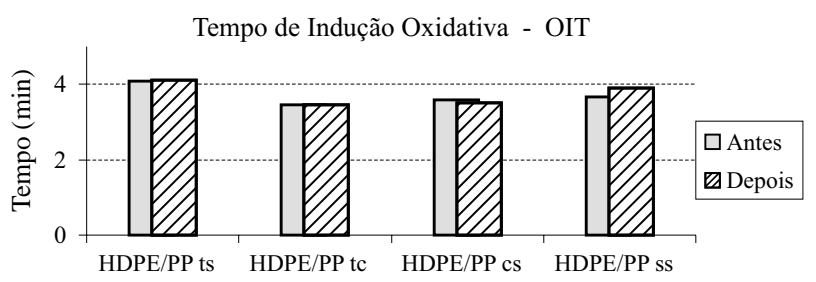

Figura 2. Gráfico dos valores de tempo de indução oxidativa das amostras de HDPE/PP, antes e após o reprocessamento.

indução oxidativa das amostras com e sem rótulos e tampas (HDPE/PP tc e HDPE/PP ts, respectivamente).

As análises da influência dos resíduos de adesivo e soda cáustica, oriundos do processo de lavagem no PET, estão apresentadas nas tabelas 5 e 6 e nas figuras 3 e 4 . Os ensaios utilizados foram medidas de viscosidade intrínseca e titulação de grupos finais carboxílicos.

Pode-se verificar que o resíduo de garrafas de PET possui viscosidade intrínseca ainda dentro do intervalo correspondente ao PET grau garrafa $(0,70-0,85 \mathrm{dL} / \mathrm{g})$. Dados estes confirmados pelo baixo valor de grupos finais carboxílicos obtidos durante a titulação. Durante o reprocessamento, a queda no valor de viscosidade intrínseca mostrouse apenas um pouco acima do PET virgem $(\Delta \eta=0,02)$, indicando provavelmente o baixo ní-

Tabela 5. Valores de viscosidade intrínseca das amostras de PET antes e após o reprocessamento.

\begin{tabular}{ccc}
\hline \multirow{2}{*}{ Amostra } & \multicolumn{2}{c}{ Viscosidade Intrínseca $(\mathbf{d L} / \mathbf{g})$} \\
\cline { 2 - 3 } & $\begin{array}{c}\text { Antes do } \\
\text { processamento }\end{array}$ & $\begin{array}{c}\text { Depois do } \\
\text { processamento }\end{array}$ \\
\hline PET cc & 0,76 & 0.71 \\
PET pc & 0.76 & 0.71 \\
PET sc & 0,76 & 0.71 \\
PET cs & 0.75 & 0.71 \\
\hline
\end{tabular}


Tabela 6. Dados da concentração de grupos finais carboxílicos das amostras de PET antes e após o reprocessamento, obtidos a partir do método de Pohl.

\begin{tabular}{ccc}
\hline \multirow{2}{*}{ Amostra } & \multicolumn{2}{c}{$[\mathrm{COOH}]\left(\mathrm{eq} / 10^{6} \mathrm{~g}\right)$} \\
\cline { 2 - 3 } & $\begin{array}{c}\text { Antes do } \\
\text { processamento }\end{array}$ & $\begin{array}{c}\text { Depois do } \\
\text { processamento }\end{array}$ \\
\hline PET cc & 31.45 & 38.63 \\
PET pc & 26.50 & 36.74 \\
PET sc & 25.62 & 34.61 \\
PET cs & 27.32 & 33.41 \\
\hline
\end{tabular}

vel de contaminação dos resíduos e o bom nível de secagem do material.

Nos dados de viscosidade intrínseca notou-se ainda uma leve influência catalítica do resíduo de adesivo, pois a amostra sem resíduo de adesivo foi a única que apresentou um decaimento menor no valor da mesma. Sua viscosidade intrínseca inicial inferior pode ser devido à amostragem das garrafas de PET ter sido diferenciada, pois escolheu-se apenas as garrafas com rótulo tipo "hotmelt".

Nos dados de titulação observou-se uma dispersão na quantidade inicial de grupos finais carboxílicos entre as amostras. Esta variação pode ser devido a variações experimentais durante a análise, uma vez que o método apresenta-se sensível a variações de temperatura e tempo. Desta forma, estas mesmas amostras estão sendo caracterizadas, em duplicata, em termos de peso molecular e de polidispersividade, através de Cromatogafia de Permeação em Gel para confrontação de dados.

Já a influência do resíduo de soda cáustica após 5 minutos de enxágüe, praticamente não interferiu de forma catalítica após o reprocessamento. Porém é provável que a remoção do resíduo de soda caústica juntamente com o de adesivo tenha um efeito mais pronunciado.

Finalmente, a não variação no nível de degradação entre diferentes condições de lavagem pode

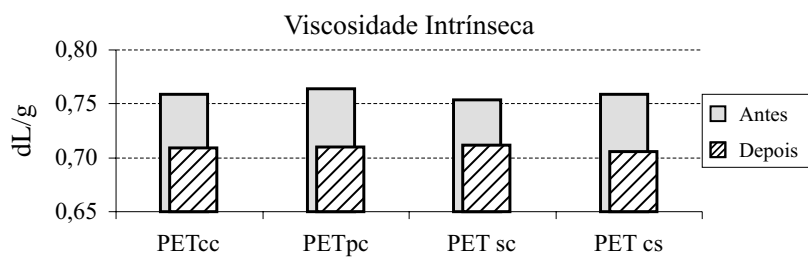

Figura 3. Gráfico da viscosidade intrínseca das diversas amostras de PET, antes e após o reprocessamento.

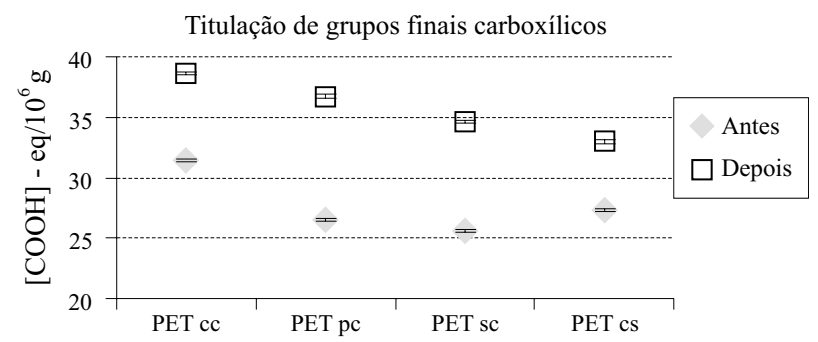

Figura 4. Gráfico do número de finais carboxílicos das diversas amostras de PET, antes e após o reprocessamento.

ser resultado da não sensibilidade dos métodos utilizados para as caracterizações.

\section{Conclusão}

Neste trabalho pode-se concluir que, apesar da diversidade dos resíduos de poliolefinas, é possível caracterizá-lo através de uma boa homogeneização prévia. Os resíduos de LDPE e PP das tampas e rótulos, mesmo apresentando uma estabilidade térmica inferior, nas proporções encontradas, não agem deteriorando a estabilidade termooxidativa do HDPE. Além do que, a presença de HDPE reprocessado a quase $20 \%$ do total, praticamente não interferiu de forma catalítica durante o reprocessamento. De acordo com estes dados, a estabilização prévia do HDPE/ PP de resíduo urbano é praticamente desnecessária antes do reprocessamento.

Para os resíduos de PET, foi possível observar a importância da remoção do resíduo de adesivo, no processo de degradação deste polímero. No entanto, a retirada deste resíduo durante a lavagem implica em custo adicional nesta etapa, uma vez que seria necessário incrementar seu intervalo de tempo. Como sua influência numa amostra com secagem bem sucedida não foi catalítica, um processo de lavagem com apenas 5 minutos pode ser vantajoso.

Quanto ao resíduo de $\mathrm{NaOH}$ após 5 min de enxágüe pode-se considerá-lo praticamente desprezível, não havendo uma melhora significativa de desempenho através de um tempo de enxágüe adicional de $5 \mathrm{~min}$. Quanto à severidade da etapa de lavagem (temperatura e aditivos de limpeza), pode-se concluir que, sob as concentrações de aditivo utilizadas, o polímero praticamente não degradou. Nem mesmo o incremento de $10 \mathrm{~min}$ no tempo de lavagem a $80{ }^{\circ} \mathrm{C}$ para remoção parcial do resíduo de adesivo, foi crítico no incremento de grupos finais carboxílicos. 
Por último, é importante enfatizar que as conclusões acima estão baseadas nos resultados obtidos com métodos de análise que podem não estar detectando degradação em nível molecular como, por exemplo, variação na estrutura e no peso molecular e sua distribuição.

\section{Agradecimentos}

Os autores agradecem ao MCT/PADCTIII/ $\mathrm{CNPq}$, agente financiador deste projeto, incluindo a Bolsa de Mestrado.

\section{Referências Bibliográfica}

1. Agnelli, J. A. M. - Degradação, estabilização e envelhecimento de polímeros. São Carlos: ABPol, 1997. p. 196-204 Apostila curso ABPol, p.115 (1997).
2. Paul, D. R.; Keskkula, H. - Encyclopedia of the polymer science and engineering New York: Jonh Wiley, v. 4 (1986).

3. Cardi, N., et al. - Chain extension of recycled poly(ethylene terephthalate) with 2,2'bis(2-oxazoline). Jounal of Applied Polymer Science, v. 50, p. 1501-1509 (1993).

4. Paci, M., La Mantia, F. P. - Competition between degradation and chain extension during processing of reclimed poly(ethylene terephthalate). Polymer Degradation and Stability, v.61, p. 417-420 (1998).

5. Mancini, S. D., Zanin, M. - (submetido a publicação).

6. Pohl, H. A. - Determination of carboxyl end groups in a polyester, polyethylene terefhthalate. Analytical Chemistry, v. 26, n.10, p. 1614-1616 (1954). 